\title{
Estudios curriculares y pertinencia social, un balance de la cuestión
}

\section{Curricular studies and social pertinence, a balance of the question}

pp. $9-20$

José Benito Garzón Montenegro ${ }^{1}$

REC: $19 / 10 / 2019$

DiANA Vinasco MARTÍNEZ ${ }^{2}$

ACEP: $19 / 05 / 2021$

\section{Resumen}

En el presente artículo se realiza un acercamiento a los conceptos de perspectivas curriculares y pertinencia social, a partir de un análisis documental que busca rastrear sus significados y la forma en que estos han cambiado en el tiempo, a partir de la aplicación que se les ha dado en la educación superior. Dichos planteamientos hacen parte de la revisión teórica para el proyecto "Perspectivas curriculares y pertinencia social de los programas académicos de licenciatura en la Universidad Católica Lumen Gentium y su aporte a la propuesta pedagógica del Capítulo Suroccidente de Ascofade al Ministerio de Educación Nacional", donde se pretende analizar la forma en que estas cuestiones vienen siendo abordadas en los procesos de formación de formadores. Se propone, a partir del análisis de diversos autores y autoras, la ampliación del significado de currículo, como forma de superar una concepción que lo limita a la planificación de cursos; luego se describe la evolución del concepto de pertinencia social y la forma en que se ha incorporado al ámbito educativo; finalmente se analizan propuestas de vinculación de estas dos nociones y se brindan algunas conclusiones al respecto, en las que se resalta la disociación entre los diferentes niveles curriculares (macromeso-micro), lo que impacta de forma directa a la manera de comprender y asumir la pertinencia social del currículo.

Palabras clave: perspectiva curricular, pertinencia social, educación superior, vinculación universidad-sociedad.

1. Doctor en Humanidades, Universidad del Valle. Doctor en Économies, Espaces, Sociétés, Civilisations, Universidad Paris 7 - Denis Diderot. Decano, Facultad de Educación, Unicatólica. Grupo de investigación Educarte. Correo electrónico: decaeducación@unicatolica. edu.co - Orcid: http:// orcid.org/ 0000-0002-5629-0401

2. Magister en Estudios Sociales y Políticos, Universidad Icesi. Docente e investigadora, programa de Licenciatura en Ciencias Sociales, Unicatólica. Correo electrónico: dvinasco@unicatólica.edu.co - Orcid: http:// orcid.org/ 0000-0002-0580-1160 


\section{Abstract}

This article makes an approach to the concepts of curricular perspectives and social relevance, from a documentary analysis that seeks to trace their meanings and the way in which they have changed over time, based on the application they have been given in higher education. These approaches are part of the theoretical review for the project "Curricular perspectives and social relevance of the academic programs of bachelor's degree at the Fundación Universitaria Católica Lumen Gentium and its contribution to the pedagogical proposal of the Southwest Chapter of Ascofade to the Ministerio de Educación Nacional", which aims to analyze the way in which these issues have been addressed in the training of trainers processes. It is proposed, based on the analysis of various authors, the expansion of the meaning of the curriculum, as a way of overcoming a conception that limits it to course planning; Then the evolution of the concept of social relevance and the way in which it has been incorporated into the educational field are described; Finally, proposals for linking these two notions are analyzed and some conclusions are provided in this regard, in which the dissociation between the different curricular levels (macro; meso; micro) is highlighted, which directly impacts the way of understanding and assume the social relevance of the curriculum.

Keywords: curricular perspective, social relevance, higher education, universitysociety bonding.

\section{Introducción}

Toda perspectiva curricular está relacionada con una determinada forma de racionalidad, es decir, con concepciones curriculares particulares vinculadas a formas específicas de concebir y asumir la educación en relación con unas expectativas y proyecciones de formación deseadas, las mismas, que son generadas o compartidas por aquellos grupos sociales dominantes. Dichas perspectivas se soportan en ciertas formas de representación y valoración que le otorgan sentido-significado y le confieren identidad a los procesos curriculares desarrollados en contextos educativos determinados, sirviéndole de soporte y sustento teórico y práctico, cuando menos, a los programas de licenciatura.

Las reformas a la educación superior que se vienen dando desde finales del siglo XX en el mundo, incluyendo a América Latina y en particular a Colombia, se basan en un modelo de internacionalización de la educación superior promovido desde la lógica de la globalización, las sociedades de la información y la llegada de las nuevas tecnologías de la información y la comunicación (TIC), a través de organismos internacionales como la OCDE, la Unesco, el Banco Mundial, el BID, etc., que impulsan la idea de incrementar la calidad de los programas académicos mediante un trabajo académico cooperativo suprainstitucional que forme al profesional para desempeñarse laboralmente en un mundo cada vez más interdependiente y competitivo.

El propósito de homogeneizar los currículos universitarios se concreta en la Declaración de Bolonia 1 para Europa y se introduce a América Latina a través del Proyecto Alfa Tuning y las diferentes declaraciones regionales realizadas sobre educación superior, dentro de una agenda globalmente estructurada para la educación, cuyo propósito es generar una innovación social y educativa en la región mediante la "modernización de los programas de estudio" con el fin de atender la "ineficiencia" en la formación profesional de que adolece la educación superior latinoamericana 
y potenciar mundialmente el proceso de Bolonia.

En el presente documento se analiza el desarrollo de los estudios sobre currículo y cómo se han relacionado con los debates sobre la pertinencia social de la educación superior. Teniendo en cuenta que la pertinencia social es el vínculo que se establece entre discurso y acción, y hace referencia a las relaciones que establece la universidad con la sociedad, como institución inserta en un contexto determinado; resulta central comprender el concepto de currículo y las dimensiones que pueden estar implicadas en él, como forma de entender su vínculo con la pertinencia social.

Lo anterior implicaría pensarse el currículo como un conjunto de componentes indisociables en sus niveles macro, meso y micro, tanto desde la práctica educativa como desde el campo de la investigación curricular. Por ello, la realización de un balance de la cuestión sobre los estudios curriculares en Colombia supone analizar los retos que dicho campo afronta, y uno de ellos, como ya se ha señalado, se relaciona con las tensiones entre sus distintos niveles y la forma en que estas han sido abordadas en las investigaciones sobre el tema.

En este sentido, asociamos lo macrocurricular con las políticas públicas nacionales e internacionales; lo mesocurricular con las políticas de cada institución respecto a la fundamentación y estructuración de sus planes de estudio; y lo microcurricular con la forma en que lo planificado en los dos niveles anteriores es llevado a la práctica en el desarrollo de las actividades de enseñanza-aprendizaje. Con ello se pretende ubicar las investigaciones de acuerdo con el relacionamiento que proponen entre los diferentes niveles del currículo y la perspectiva o enfoque curricular en el que se inscriben.

\section{Qué es el currículo}

Cada vez más se hace más complejo definir y conceptualizar el currículo. Son dos razones fundamentales la que originan dicha situación: la primera, ligada a su carácter polisémico, ya que existen aportes y debates establecidos por los diferentes enfoques académicos desarrollados en la historia reciente (p.e.: técnico / racional / academicista, interpretativo / simbólico / empirista / conceptualista y socio-crítico / reconceptualista); la segunda tiene que ver con las demandas que socialmente se les hacen a las instituciones educativas, ligadas a las vertiginosas transformaciones del mundo actual (dinámicas socioeconómicas globales, inciertas y cambiantes; dinámicas familiares que limitan el vínculo social, la socialización, la estabilidad emocional y la formación axiológica de los sujetos; descohesión social, para señalar las más preponderantes), las mismas que suelen ser tramitadas por los Estados a manera de regulación prescriptiva.

En el caso colombiano la concepción curricular que ha primado en el tiempo reciente es la asociada con el carácter técnico de la misma, a manera de "planificación" (Montoya, 2016), en particular de los contenidos cognitivos que deben recibir quienes emprenden un proceso de formación formal, es decir, con una especie de instrumentalización de las prácticas educativas en las instituciones escolares de todo nivel. Dicha concepción está en sintonía y favorece las agendas tecnocráticas de los gobiernos de turno, en la lógica del alineamiento internacional; lo que en últimas se ha traducido en un opacamiento o disminución de la acción pedagógica de los maestros y de las instituciones educativas de todo nivel, al punto de reducir sus labores a impartir asignaturas fragmentadas y secuencializadas de manera lineal y rígida, que permitan la 
adhesión de conocimientos al estudiante, dejando de lado otras dimensiones fundamentales en la formación de los mismos.

Dicha versión reduccionista del currículo, y en particular la disociación con la pedagogía, ha marcado una suerte de oposición entre dichas categorías. Así el currículo fue equiparado con la instrucción (con todo lo que esta implica) mientras que la pedagogía se asimila con la enseñanza-aprendizaje. El efecto inmediato, y que aún se mantiene, es el alejamiento de los maestros a indagar por el currículo, tras la prelación a lo pedagógico, lo que ha repercutido, al decir de Juny Montoya (2016), en un ensombrecimiento del currículo como campo de estudio. Tal efecto ha limitado la discusión sobre el currículo, a la vez que le ha disminuido su importancia como objeto de investigación, como ya se advirtió, al situar al currículo como una noción meramente prescriptiva y rígida, sin tener en cuenta otras maneras de interpretarlo, diseñarlo y asumirlo.

Por lo anterior, lo curricular en el país se ha convertido en un campo ávido de discusión, pero que no ha dejado de operar, cuyo diseño ha estado centrado en las dimensiones cognitivas, prácticas y técnicas (en el mejor de los casos); aspectos ligados fundamentalmente a las disposiciones del Estado, que se reflejan en los sistemas de evaluación dispuestos para ello. Así, se han dejado de lado otras dimensiones de la formación del ser humano como lo emocional, lo espiritual, lo comunicacional y lo axiológico, con lo cual el ámbito de la formación educativa se circunscribe al sabery al hacer, dejando de lado el ser.

Ampliar la noción de currículo impone algunos retos fundamentales para los procesos educativos, ya que obliga a trascender la idea de la planificación inflexible y descontextualizada, para tener en cuenta las búsquedas, necesidades y propósitos de los estudiantes, así como las experiencias y conocimientos/ saberes de los profesores, todo ello en el marco de los desarrollos de la sociedad y las ciencias, así como de las condiciones de cada una de las instituciones educativas. Esta concepción busca superar las nociones que ven al currículo solo como la planeación técnica de los procesos educativos rígidamente secuencializados, así como aquellas que lo asumen como el mero conjunto de las experiencias educativas distantes a la planeación.

Lo anterior solo es posible si se involucra a la pedagogía como fundamento mismo del currículo, de allí el reto inmediato si se quieren superar los enfoques reduccionistas antes planteados, pues, como lo propone Castro (2004), el currículo se compone de factores que siempre están presentes en todas las dinámicas de enseñanza-aprendizaje, tales como propósitos, contenidos, metodologías, recursos didácticos, clima educativo, etc.; por lo cual, en vez de una disociación, lo que observa es al currículo como sinónimo de la dinámica de enseñanza-aprendizaje y a la pedagogía como la disciplina que circunscribe el campo de acción del currículo.

De esta forma, aunque las experiencias educativas no podrían ser planificadas en su totalidad, los elementos involucrados y los escenarios en los que se desarrolla la práctica educativa sí pueden ser dispuestos, instalados y gestionados en los planes de estudio de cada institución educativa, pues estos constituirán un marco de referencia para desarrollar la vivencia de enseñanza-aprendizaje, de acuerdo con los parámetros determinados por el modelo pedagógico establecido previamente, con base en el contexto en que se desarrolla el aprendizaje y en las necesidades de los sujetos que la componen, así como de las comunidades con las que se interactúa. 
Con lo cual lo curricular, basado en lo pedagógico, no determinaría las experiencias de enseñanza-aprendizaje, pero sí las posibilitaría (de Zubiría, 2013).

En ese sentido, podemos definir el currículo como un modo de organizar una serie de prácticas pedagógicas en correspondencia con un determinado modelo pedagógico, para lo cual se tienen en cuenta aspectos relacionados con las formas de organización de los contenidos y su dosificación en los procesos de enseñanza y aprendizaje, en correspondencia clara con la función social de la institución que lo imparte. Una perspectiva curricular pensada desde lo pedagógico, y siempre como un lugar posible y deseable de alcanzar (Stenhouse, 1991; Elliot, 1991), permite posicionar la pertinencia social de la institución como elemento fundamental del desarrollo integral del ser humano en comunidad (lo que incluye la enseñanza-aprendizaje), reconociendo las necesidades y urgencias de la sociedad, así como las de cada sujeto en específico.

En ese orden de ideas, si el currículo debe pensarse y disponerse para el desarrollo de la persona a través de lo social, dicho currículo no estará determinado únicamente por las labores formativas de carácter cognitivo en el aula, sino por el encuentro y la interacción con los distintos sujetos de la comunidad donde hace presencia la institución educativa.

Ello implica, además, tener en cuenta como componentes de lo curricular elementos y procesos que están por fuera del plan de estudios y de las asignaturas, tales como la proyección social, la investigación y los procesos culturales que involucren a toda la comunidad académica en relación con los contextos específicos.

\section{El concepto de pertinencia social en la educación superior}

Lo anterior impone otro reto a la hora de realizar un diseño curricular, y es el asumir la tensión existente entre las políticas educativas, que a manera de normatividad y planes indica el Ministerio de Educación Nacional (MEN), con los contextos institucionales, Iocales y regionales, así como con los saberes y conocimientos con que cuentan los docentes, estudiantes y comunidades intervinientes en los actos educativos, con la clara conciencia de que el currículo previene componentes y determinaciones de muy diverso cuño: pedagógicas, políticas, prácticas administrativas, etc. (Lafrancesco, 2003).

Es allí cuando resulta necesario analizar la relación universidad-sociedad, a través del grado de correspondencia existente entre las necesidades sociales e individuales que se pretende satisfacer con la educación y lo que realmente se llega a alcanzar, es decir, la pertinencia social de la educación. Dicho concepto es definido por la Unesco en el "Documento de Política para el Cambio y Desarrollo en la Educación Superior", como el papel que desempeña el sistema de enseñanza a través de las instituciones educativas respecto a la sociedad y lo que esta última espera de la educación:

La pertinencia debe pues abarcar cuestiones como la democratización del acceso y mayores oportunidades en la educación superior durante las distintas fases de la vida, los vínculos con el mundo del trabajo y las responsabilidades de la educación superior con respecto al sistema educativo en su conjunto. No menos importante es la participación de la comunidad de la educación superior en la búsqueda de soluciones a problemas humanos apremiantes como la demografía, el medio ambiente, la paz y el entendimiento internacional, la democracia y los derechos humanos. 
La mejor manifestación de la pertinencia de la educación superior tal vez sea la variedad de "servicios docentes" que presta a la sociedad. (Unesco, 1995, p. 29)

Para Naidorf et al. (2007), el concepto de pertinencia social debe ser analizado desde una perspectiva crítica, pues la bibliografía académica ha tendido a presentarla como unívoca, ahistórica y neutral, desconociendo su surgimiento y aplicación a partir de un contexto histórico y político específico. Dicho surgimiento es ubicado, por las mismas autoras, en el marco de la vinculación de la universidad con el mundo empresarial, propiciado por políticas económicas globalizantes e instrumentalizantes de la educación en Latinoamérica, aproximadamente a mediados de los años noventa del siglo pasado.

De esta manera, la pertinencia social - noción propia del ámbito organizacionalpasa a reemplazar cuestiones como la misión o la función social de las instituciones de educación superior, y se convierte en un concepto central para el análisis del ámbito educativo y de las relaciones universidad-sociedad, a través de planes de estudio y actividades de extensión que vinculen la institución educativa con las necesidades productivas y empresariales de la sociedad. Sin embargo, dicha definición cambia "cuando se hacen alusiones a la Sociedad como el espacio de las mayorías que son excluidas del sistema" (Naidorf et al., 2007, p. 26).

Lo anterior supone tener en cuenta que la autonomía de los sujetos, al considerar lo que para ellos es o no pertinente, pues, como señala Naishat (2003), lo que para gran parte de una sociedad puede ser impertinente, para otra parte puede estar dotado de sentido, si se tiene en cuenta que la pertinencia de la educación también podría suponer la crítica a lo establecido y sus posibilidades de cambio. En ese sentido, lo que el autor denomina "impertinencia epistémica" supone la formación de sujetos críticos y autónomos capaces de analizar si la sociedad en la que se encuentran insertos se corresponde con su visión de lo que debería ser pertinente. Así, la pertinencia institucional de la universidad pasaría por defender el principio de impertinencia epistémica como condición de libertad y pensamiento crítico (Naidorf et al., 2007).

A pesar de estas críticas, es claro que la pertinencia social hace referencia a las relaciones que establece la universidad con la sociedad, como institución inserta en un contexto determinado. Dichas críticas no suponen precisamente la eliminación del concepto de pertinencia social para la proyección universitaria, sino la adecuada comprensión del concepto y el análisis de las dimensiones que pueden estar implicadas en él, como forma de sacarlo del ámbito empresarial y productivo, transformándolo en categoría útil para otros aspectos en los que la universidad y la sociedad se vinculan.

\section{Posturas pertinentes sobre el currículo}

En su tesis doctoral sobre el proceso de configuración del campo curricular en Colombia durante el siglo XX, señala Fernández (2016) que el nivel de educación superior se ha convertido en el principal productor de investigaciones sobre el currículo y que dichos trabajos se han centrado, principalmente, en la relación entre dimensión institucional y sociedad.

Trabajos como los realizados por Malagón $(2004,2006,2008)$ donde reflexiona sobre el currículo como el puente que permite la interactividad entre la universidad y la sociedad. 
Dicho concepto es definido por el autor como un proceso, mediante el cual se organiza, se selecciona, se produce y se reproduce la cultura, pues además de la formación científica y técnica, el propósito de la universidad pasa por la intervención en el entorno a través de la formación integral que permita procesos de cambio. Así mismo, argumenta que las mayores posibilidades de ofrecer una formación integral se basan en la capacidad de una institución educativa para intervenir en procesos sociales, interactuar de forma crítica con el entorno y flexibilizar sus procesos educativos.

En la misma línea de análisis se enfoca Álvarez (2010), quien define el currículo como el aspecto que media la relación entre el proyecto educativo de la institución y las prácticas educativas llevadas a cabo por docentes y estudiantes, y que está atravesado por las condiciones sociohistóricas de su contexto. Al igual que Malagón, afirma que el currículo es un proceso, y añade que es una guía provisional sobre la que debe reflexionar constantemente - luego de ser llevada a la práctica - toda la comunidad académica, pues lo curricular permite articular la práctica educativo-pedagógica desde un sentido formativo.

Sin embargo, afirma que lo regularmente conocido por directivos y profesores son los niveles macro (políticas gubernamentales) y micro (programación de asignaturas) de los diseños curriculares, pero lo que muchas veces se ignora es el nivel en el cual se planifican los programas de estudio, conectando los otros dos niveles, lo cual supone ignorar "cuánto de lo escrito, de verdad, obedece a las comprensiones de sus autores y gestores, u obedece a su fantasía, a la cultura libresca y memorística; cuánto ocurre en la práctica, y cuánto de verdad produce el efecto que se espera" (Álvarez, 2010, p. 83).
Para el autor, el problema radica en la falta de acercamiento que muchos docentes suelen tener a la creación de las propuestas curriculares, y que en últimas significa un alejamiento de los procesos de reflexión, evaluación, diseño y mejora continua del currículo, en los que deberían estar involucrados, independiente de las disciplinas que manejen, pues dicho acercamiento constituye una forma de que los planteamientos mesocurriculares tengan mayor posibilidad de conexión con el nivel microcurricular; es decir, sean realmente puestos a prueba en el aula de clases.

Aunque estos dos autores tienen la pretensión de describir y detallar los significados que las instituciones educativas otorgan al desarrollo curricular y cómo responden a los intereses de la sociedad en la que están insertas, no es clara la forma en que dichos aspectos se llevan a la práctica en el aula de clase, ni cómo se vinculan con las políticas estatales que rigen la materia. En el caso de la reflexión propuesta por Álvarez, cabría preguntarse cómo vincular reflexiones constantes en correspondencia con el contexto, conservando los lineamientos rígidos del MEN. El mismo reto se impone en la flexibilización de procesos educativos planteada por Malagón como componente central de la pertinencia social, pues dicha flexibilidad debe pensarse desde la forma en que el Estado delinea y delimita la secuencialización y obligatoriedad de gran parte de los contenidos.

A pesar de ello, el texto de Álvarez es una apuesta que critica el papel pasivo de los docentes ante lo que la planificación curricular implica, y plantea que la clave de la vinculación entre los tres niveles curriculares está en el papel que cumplan los y las docentes en los planteamientos curriculares de las instituciones. Dicha propuesta vincula la docencia a las labores de investigación-acción sobre lo 
curricular, ya que, tal como señala Elliot (1991), lo contrario significaría aceptar las imposiciones curriculares de "expertos" que moldean técnicamente las prácticas educativas, pero que no suponen la reflexión constante sobre las mismas:

Los profesores investigadores que descuidan el estudio de las estructuras curriculares, reducen la Investigación-Acción a una forma de reaccionismo técnico dirigido a mejorar sus aptitudes técnicas. Es muy probable que esto ocurra si los profesores reflexionan aislados entre sí. El individuo aislado que se enfrentara al modo en que las estructuras curriculares modelan su práctica, se daría cuenta de su impotencia para llevar a cabo el cambio. [...] Yo afirmaría que la aparición generalizada de la Investigación-Acción colaborativa como método de evaluación curricular basado en los profesores, es una respuesta creativa al crecimiento de sistemas técnico-racionales de vigilancia de control jerárquicos sobre las prácticas de los profesores. De los rescoldos de la cultura tradicional surge el fénix de una práctica reflexiva y colaborativa para oponer una resistencia creativa a la hegemonía del tecnócrata. (Elliot, 1991, p. 56)

Lo que Elliot denomina "hegemonía del tecnócrata" supone pensar que quienes desarrollan el currículo son expertos conocedores de las políticas curriculares, de la planeación curricular y de las cuestiones administrativas de las instituciones educativas, pero que no son los mismos docentes. Es este el mismo llamado de atención de Juny Montoya, respecto a romper la separación entre pedagogía y currículo, proponiendo que los expertos en lo curricular deben ser, precisamente, quienes lo aplican en el aula.

Quintero et al. (2007) afirman que, en Colombia, a partir de la promulgación de la Ley 115 de 1994, se promovió la participación de los docentes en la construcción de procesos y materiales curriculares, impulsando con ello la investigación-acción en el país. Valdría preguntarse si, al ser desarrollados por docentes, los planteamientos curriculares sí cumplirían su papel de incidir en lo pedagógico, en lo social y se conectarían con las necesidades y expectativas de los estudiantes.

Al respecto, varias investigaciones podrían brindar luces sobre esta cuestión, pues cabe decir que, apoyando lo señalado en el párrafo anterior, en los últimos años las investigaciones sobre el currículo en Colombia sí han estado en manos de docentes, que han combinado sus labores pedagógicas con la investigación y que han planteado cuestiones sobre la aplicabilidad de la relación educación-sociedad en el currículo, es decir, si efectivamente el currículo está intentando responder a las múltiples necesidades de los estudiantes y de la sociedad.

Aristizábal et al. (2005) plantean una aproximación crítica a dicho concepto a partir de cuatro preguntas, una de las cuales es: " $i A$ qué problema social, económico, político 0 educacional trata de responder el currículo en la actualidad? 0 por el contrario: ¿se afectan los currículos por las situaciones sociales, políticas, económicas y culturales?" (p. 5). Tratando de dar respuesta a esta pregunta, afirman que lo único claro es que la universidad ha asumido el deber de formar profesionales para que se ubiquen laboralmente, y aunque es necesario que contribuya al desarrollo de otras competencias relacionadas con lo social y ético, los cambios que ha sufrido la educación en Latinoamérica en las últimas décadas no han tenido a los docentes como protagonistas de dichos cambios, pues la fuerza de la "curricularización" de la educación terminó por ocultar las verdaderas dimensiones políticas, económicas e históricas del currículo (Aristizábal et al., 2005, p. 8). 
Es decir que, independiente de su involucramiento en el desarrollo curricular, la importancia otorgada a los saberes técnicos, a la educación para el trabajo, a lo práctico e instrumental, ha disminuido el rol político de los docentes en el aula, y aunque se involucren en los planteamientos curriculares, dichos planteamientos deben estar alineados con intereses económicos y políticos más amplios.

Varios trabajos plantean la necesidad de que el currículo reconozca, involucre y se adapte al contexto en que está inmersa la institución. Jacinto y Heras (2010), por ejemplo, afirman que el diseño curricular no puede ser dejado a la "simple intuición de los docentes", pues lo más importante es tener muy claro, qué tipo de sujetos se quiere formar y cómo se puede lograr este objetivo. En el mismo sentido, señalan Quintero et al. (2007), el currículo es y será un proyecto intencionado que reproduce el orden cultural existente en la sociedad en que se encuentra inserta la institución educativa y, por ello, los desafíos del mundo actual, planteados por la globalización y por los cambios tecnológicos, exigen cambios acelerados a las universidades. Pero ¿cuál es ese tipo de sujeto?, ¿de qué tipo de proyecto cultural estamos hablando?, ¿cuáles son esos cambios acelerados que deben hacer las universidades?

\section{Acuerdos (directrices, disposiciones) internacionales sobre el currículo}

Respecto a lo anterior, y tratando de explorar qué tipo de proyecto educativo estaría demandando la sociedad actual, conviene analizar el documento publicado en 2016 por la Unesco-OIE, denominado ¿Qué hace a un Currículo de calidad?, que afirma como propósito determinar los parámetros que garanticen la calidad curricular, permitiendo concretizar el objetivo en mención, pues se considera al currículo como esencial para lograr una educación pertinente, integral, inclusiva y, en consecuencia, de calidad. Así mismo, más que determinar de manera prescriptiva lo que debería ser un currículo, pretende ofrecer un marco conceptual que brinde criterios para evaluar la calidad de los currículos ya existentes 0 en proceso de elaboración.

Para la Unesco (2016), el currículo cumple un papel esencial en el vínculo entre el desarrollo y la educación, pues articula y proporciona aspectos clave de los procesos educativos, como la garantía de inclusión y equidad, la estructura necesaria para impartir un aprendizaje de calidad, las competencias que permiten un aprendizaje a lo largo de la vida, y la satisfacción de necesidades en un sentido holístico.

Por ello, propone entender el currículo como un "acuerdo político y social", en el cual se refleja la visión de una sociedad, teniendo en cuenta necesidades y expectativas que van desde el plano local hasta el global; dicha visión incide tanto en los propósitos y objetivos de la educación como en las formas de organizar los procesos de enseñanza, aprendizaje y evaluación.

En este sentido, afirma el documento que los responsables de elaborar los currículos deben responder a interrogantes fundamentales como: ¿Qué conocimientos, capacidades y valores deberíamos incluir en nuestro currículo? ¿La adquisición y el desarrollo de esos conocimientos, capacidades y valores, y de las capacidades y competencias conexas, permitirían que nuestros jóvenes pudieran llevar una vida productiva y significativa? ¿De qué manera podemos hacer que el aprendizaje sea pertinente e interesante para los estudiantes? 
En la formulación de estas preguntas, señala el mismo documento, subyace el concepto de calidad como eje fundamental para los cambios que se deban hacer a un currículo. Su nivel de presencia en una propuesta curricular podría determinarse a través de una serie de indicadores, tales como la pertinencia, la coherencia, la viabilidad, la sostenibilidad y la eficacia.

Asumir la propuesta de la Unesco (2016) significaría que plantear una propuesta curricular, implica tener en cuenta lo que la sociedad demanda y ello no pasaría precisamente por lo que la universidad debería representar en la sociedad, pues si el acuerdo político, implícito o explícito, es que la educación debe estar encaminada a la productividad y alinearse con las demandas económicas y empresariales de un mundo globalizado, esto supondría un acuerdo político que debilitaría la autonomía y el papel social de la universidad (Beltrán y Torres, 2004).

En el mismo sentido, señala Misas (2004) que una sobreadaptación de la universidad a lo que la sociedad demande, significaría la pérdida de sus características centrales: "Si la universidad se sobreadapta, como quieren paradójicamente tanto el mundo neoliberal como el mundo radical que pretenden que se acomode a las condiciones del momento, perdería su esencia y su razón de ser" (p. 51).

La universidad debería, entonces, cuestionar si el nivel de pertinencia de su perspectiva curricular debe medirse de acuerdo con el grado de integración con las demandas inmediatas de la sociedad, o si, además de este aspecto, debería suponer el cuestionamiento crítico a estas demandas y qué tanto están contribuyendo a la integración de todos los actores sociales. La pertinencia de lo curricular, vista como el grado de correspondencia entre lo que la universidad propone y lo que la sociedad requiere, debe pasar también por el análisis, cuestionamiento y resignificación de estos requerimientos, como forma de contribuir no solo a lo que la sociedad demanda en su momento, sino a repensar la forma en que estas demandas contribuyan a una sociedad más inclusiva y plural.

\section{Hallazgos preliminares}

Una primera hipótesis respecto al campo curricular en Colombia, podría sugerir que la disociación entre lo curricular y lo pedagógico ha estado sustentada en la investigación misma, debido a que los trabajos que han abordado dicho campo, se han centrado, por un lado, en los aspectos macro (políticas públicas) y meso (diseño institucional de programas de estudio). Igualmente, quienes se han interesado en estudiar lo micro, es decir, el diseño del desarrollo mismo de los cursos, lo han hecho sin tener en cuenta su vínculo con los aspectos macrocurriculares.

Así, aunque algunos niveles han intentado la asociación entre los niveles macro y micro, y otros lo han hecho con los niveles meso y micro, prevalecen los análisis fragmentados, dejando de lado la conexión de los tres niveles, que sería la forma de poner a prueba el alcance y la pertinencia social del currículo. En este sentido, se trata de un tema que compete no solo a los definidores de la política educativa, sino a la manera de concepción y construcción de la misma, pues se evidencia que en el fondo de la disociación señalada está la escasa o nula participación de sectores sociales clave en la formulación, diseño y gestión de la política educativa, para lo cual se requiere de voluntad y disposición de todas las partes para superar estas dificultades y avanzar en el abordaje de estos temas densos. 
En cuanto a la pertinencia social, su utilización conceptual ha sido más un argumento para el diseño macrocurricular que una apuesta por la formación de sujetos que aporten a la sociedad de manera crítica y ética, pues ha terminado por obedecer a condiciones e intereses no explícitos indicados por organismos multilaterales. Así se evidencia que, en general, la pertinencia social ha sido también limitada, debido a que no ha habido una consulta de las expectativas de las comunidades, e incluso, de los intereses políticos, axiológicos y culturales de los y las estudiantes; dictando más bien una serie de directrices, comúnmente aceptadas, sobre la adecuada inserción en el mercado laboral, lo que además se evidencia en las formas y contenidos de los que son objeto de evaluación para poder egresar de un programa profesional, v.g. Pruebas Saber Pro.

Las discusiones han estado centradas en la flexibilidad o no del currículo, en los desarrollos de las dimensiones del currículo (esencialmente lo cognitivo y lo práctico, a propósito de la formación por competencias, tras una limitada comprensión de estas últimas) y la pregunta por la pertinencia social ha estado relegada continuamente, en especial en las escalas meso y microcurricular. A lo anterior se le suman las preocupaciones por concretar o responder a las solicitudes que de forma permanente hace el MEN en relación con la formación disciplinar en algunas áreas de conocimiento, lineamientos que no han logrado ser estables y duraderos, lo que ha incidido de forma relevante en el diseño e implementación de planes de estudio postulados a validación para la obtención de registros calificados 0 acreditaciones de calidad ante las instancias del MEN dispuestas para ello.

En fin, los hallazgos aquí esbozados dejan una serie de retos, a diferentes escalas, para cada una de las instancias encargadas de pensar, diseñar y gestionar el currículo, a partir de la pregunta por la pertinencia curricular del mismo. Se espera que tanto el MEN como las asociaciones y agremiaciones que reúnen a las unidades académicas que ofertan programas de formación profesional, así como a las direcciones académico-administrativas de los programas y el mismo profesorado, asuman un papel más coordinado y reflexivo sobre el desarrollo de los procesos académicos, para lo que tienen que poner de manifiesto la perspectiva curricular que les convoca, así como la forma de asumir y dar respuesta a la pertinencia social del currículo que proponen.

\section{Referencias}

Álvarez, M. (2010). Diseñar el currículo universitario: un proceso de suma complejidad. Signo y Pensamiento, 29(56), 68-85.

Aristizábal, M., Calvache, L., Castro, G. J., Fernández, A., Lozada, L., Mejía, M. E. y Zúñiga, J. 0. (2005). Aproximación crítica al concepto de currículo. Revista ieRed, 1(2), 1-12.

Beltrán, W. M. yTorres, M. R. (2015). Calidad y pertinencia del programa curricular de Sociología de la Universidad Nacional de Colombia desde la perspectiva de sus egresados. Revista Colombiana de Sociología, 38(2), 139-165.

Castro, F. (2004). Currículum y evaluación. Universidad de Bio Bio.

Elliot, J. (1991) Estudio del currículum escolar a través de la investigación interna. Revista Interuniversitaria de Formación del Profesorado, 10, 45-68. https:// bit.ly/ 3HZFk5B

Fernández, A. (2016). El proceso de configuración del campo curricular en Colombia entre 1994 y 2010: una investigación basada en la producción escrita de sus autores/as más representativos/as [Tesis de doctorado en Educación]. Universidad de Antioquia. 
Jacinto, G. y Heras, R. (2010). Elementos para el diseño curricular. En: V. García (comp.), Primer Congreso Latinoamericano de Ciencias de la Educación (pp. 130-146). Universidad Autónoma de Baja California.

Lafrancesco, G. (2003). Nuevos fundamentos para la transformación curricular. Editorial Cooperativa Magisterio.

Malagón, L. (2004). El currículo: dispositivo pedagógico para la vinculación universidad-sociedad. Revista Electrónica de la Red de Investigación Educativa, 1, 110-138.

Malagón, L. (2006). La vinculación Universidad-Sociedad desde una perspectiva social. Educación y Educadores, 9(2), 79-93.

Malagón, L. (2008). El currículo: perspectivas para su interpretación. Investigación y Educación en Enfermería, 26(2), 136-142.

Malagón, L. (2009). La pertinencia curricular: un estudio en tres programas universitarios. Educación y Educadores, 12(1), 11-27.

Misas, G. (2004). La educación superior en Colombia: análisis y estrategias para su desarrollo. Editorial Universidad Nacional.
Montoya, J. (2016). El campo de los estudios Curriculares en Colombia. Universidad de Los Andes.

Naidorf, J., Giordana, P. y Horn, M. (2007). La pertinencia social de la Universidad como categoría equívoca. Nómadas, 27, 22-33.

Naishat, F. (2003). Universidad y conocimiento: por un ethos de la im-pertinencia epistémica. Espacios de Crítica y Producción, 30, 23-40.

Quintero, J., Munévar, R. A. y Yepes, J. C. (2007). Investigación-acción y currículo; un recorrido por el mundo. Revista Latinoamericana de Estudios Educativos, 3(1), 123-142.

Stenhouse, L. (1991). Investigación y desarrollo del curriculum. Ediciones Morata.

Unesco (1995). Documento de política para el cambio y el desarrollo en la educación superior. Unesco.

Unesco-OIE (2016). ¿Qué hace a un currículo de calidad? Reflexiones en progreso № 2 sobre cuestiones fundamentales y actuales del $\mathrm{Cu}$ rrículo y el aprendizaje. Unesco.

de Zubiría, J. (2013). Cómo diseñar un currículo por competencias. Editorial Magisterio. 